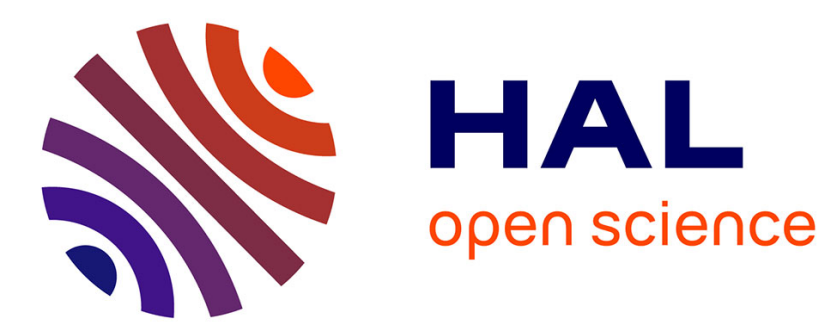

\title{
New insights into second and fourth-order direction finding for NonCircular sources
}

\author{
Anne Ferreol, Pascal Chevalier
}

\section{To cite this version:}

Anne Ferreol, Pascal Chevalier. New insights into second and fourth-order direction finding for NonCircular sources. 2014 IEEE 8th Sensor Array and Multichannel Signal Processing Workshop (SAM), Jun 2014, A Coruna, Spain. pp.465-468, 10.1109/SAM.2014.6882443 . hal-02462464

\section{HAL Id: hal-02462464 https://hal.science/hal-02462464}

Submitted on 31 Jan 2020

HAL is a multi-disciplinary open access archive for the deposit and dissemination of scientific research documents, whether they are published or not. The documents may come from teaching and research institutions in France or abroad, or from public or private research centers.
L'archive ouverte pluridisciplinaire HAL, est destinée au dépôt et à la diffusion de documents scientifiques de niveau recherche, publiés ou non, émanant des établissements d'enseignement et de recherche français ou étrangers, des laboratoires publics ou privés. 


\section{New Insights into Second and Fourth-Order Direction Finding for NonCircular Sources}

\author{
Anne Ferréol ${ }^{(1)}$ \\ (1) THALES Communications et security \\ 4, avenue des Louvresses, 92622 Gennevilliers, France \\ Email: anne.ferreol@thalesgroup.com
}

\author{
Pascal Chevalier ${ }^{(2,1)}$ \\ ${ }^{(2)}$ CNAM, CEDRIC Laboratory \\ 292 rue Saint-Martin, 75141 Paris Cedex 3, France \\ Email: pascal.chevalier@cnam.fr
}

\begin{abstract}
These last three decades, many second order (SO) and higher order (HO) high resolution (HR) direction finding (DF) methods, such as $2 q$-MUSIC $(q \geq 1)$, exploiting the information contained in the SO or HO circular (C) cumulants of the data, have been developed. However, for $2 q$ th-order noncircular (NC) sources such as $M$-PSK sources with $M \leq 2 q$, strong gains in performance may be obtained by taking into account the information contained in both $2 q$ th-order $\mathrm{C}$ and NC cumulants of the data, giving rise to NC $2 q$ th-order DF methods. Numerous NC DF methods have been developed these last fifteen years but mainly at the SO and under restrictive assumptions on the sources. The purpose of this paper is to give new insights into NC $2 q$-MUSIC methods for $1 \leq q \leq 2$ and for arbitrary NC sources.
\end{abstract}

\section{INTRODUCTION}

From the beginning of the 1980s, many SO, HR, DF methods have been developed among which the MUSIC method is the most popular [1]. To improve the performance of SO methods, in terms of resolution, robustness to modeling errors and number of sources to be processed in particular, HO HR DF methods have been developed for non-Gaussian sources from the end of the 1980s. Among these methods, extensions of MUSIC to both fourth-order (FO) and $2 q$ th-order $(q>1)$, called respectively 4-MUSIC [2] and 2q-MUSIC [3], are the most popular. These $2 q-$ MUSIC methods $(q \geq 1)$ exploit the information contained in the $2 q$ th-order circular cumulants of the data. However, for $2 q$ th-order NC sources such as $M-\mathrm{PSK}$ sources with $M \leq 2 q$, omnipresent in radio-communications, the information contained in the $2 q$ th-order circular cumulants of the data is not exhaustive and some information is also contained in the $2 q$ th-order NC cumulants of the data. In such conditions, strong gains in performance may be obtained by taking into account the information contained in all the $2 q$ thorder cumulants of the data, circular or not, giving rise to $2 q$ th-order NC DF methods.

Numerous SO NC DF methods, and NC extensions of MUSIC in particular, have been developed these last fifteen years, but under restrictive assumptions about the sources such as the assumptions of rectilinear sources [4][5] or of mixtures of rectilinear and circular sources [6]. Let us recall that a rectilinear source has a real-valued complex envelope to within a constant phase term. Only two papers [7][8] propose NC extensions of MUSIC for arbitrary sources, among which only [7] proposes a generic algorithm able to process all kind of sources with the capability to process up to $2 N-2$ rectilinear sources from $N$ antennas. On the other hand, HO NC DF methods are very scarce among which [9] and [10] propose, for rectilinear sources only, a bi-quaternion $\mathrm{NC}$ extension of 4 -MUSIC and a NC extension of $2 q$-MUSIC $(q \geq 1)$ respectively. In [11] the concept of $k$-rectilinear source has been defined as a source which can be decomposed as the sum of $k$ statistically independent rectilinear sources and the results of [10] have been extended for mixtures of $k$-rectilinear and $2 q$ th-order circular sources. More precisely two NC extensions of 2q-MUSIC, called NC1-2q-MUSIC and NC2-2q-MUSIC respectively, have been proposed in [11] for such mixtures although the latter may be used in all contexts. The first one implements a search procedure in both the phase and the direction of the sources. The second one, based on the application of [12] to the first one, limits the search to the direction only but is very costly due to the need to compute a $(q+1) \times(q+1)$ determinant for each point of the pseudospectrum.

The purpose of this paper is to give new insights into the full $(\mathrm{C}+\mathrm{NC}) 2 q$ th-order statistics of the data for $1 \leq q \leq 2$ and for arbitrary sources potentially $2 q$ th-order NC. These new insights allow to show in particular that the $N C 1-2 q$-MUSIC method developed in [11] for $k$-rectilinear sources only is in fact powerful for most of the sources (C and $\mathrm{NC}$ ) encountered in practice.

\section{MODEL AND PROBLEM FORMULATION}

\section{A. Model and extended model}

We consider an array of $N$ narrow-band (NB) sensors and we call $\mathbf{x}(t)$ the vector of complex amplitudes of the signals at the output of these sensors. Each sensor is assumed to receive the contribution of $P$ zero-mean statistically independent NB sources corrupted by a noise. Under these assumptions, the observation vector can be written as follows

$$
\mathbf{x}(t)=\sum_{i=1}^{P} \mathbf{a}\left(\Theta_{i}\right) m_{i}(t)+\mathbf{n}(t)
$$

where $\mathbf{n}(t)$ is the noise vector, assumed to be zero-mean, spatially white, circular and Gaussian, a $(\Theta)$ is the steering vector, $m_{i}(t)$ and $\Theta_{i}$ are the complex envelope and the direction of the source $i$.

NC DF methods exploit the information contained in the extended observation vector $\tilde{\mathbf{x}}(t)=\left[\begin{array}{lll}\mathbf{x}^{T}(t) & \mathbf{x}^{H}(t)\end{array}\right]^{T}$, where 
$T$ and $H$ denote transposition and conjugation-transposition respectively. From (1) we deduce that $\tilde{\mathbf{x}}(t)$ can be written as $\tilde{\mathbf{x}}(t)=\sum_{i=1}^{P} \mathbf{A}\left(\Theta_{i}\right) \mathbf{m}_{i}(t)+\tilde{\mathbf{n}}(t)=\sum_{i=1}^{P} \tilde{\mathbf{A}}\left(\Theta_{i}\right) \tilde{\mathbf{m}}_{i}(t)+\tilde{\mathbf{n}}(t)$

where $\mathbf{m}_{i}(t)=\left[\Re\left(m_{i}(t)\right) \quad \Im\left(m_{i}(t)\right)\right]^{T}, \quad \tilde{\mathbf{m}}_{i}(t)=$ $\left[\begin{array}{ll}m_{i}(t) & m_{i}^{*}(t)\end{array}\right]^{T}, *$ means complex conjugate, $\tilde{\mathbf{n}}(t)=$ $\left[\begin{array}{ll}\mathbf{n}^{T}(t) & \mathbf{n}^{H}(t)\end{array}\right]^{T}, \tilde{\mathbf{A}}(\Theta)=\left[\begin{array}{ll}\mathbf{a}_{1}(\Theta) & \mathbf{a}_{2}(\Theta)\end{array}\right]$ where $\mathbf{a}_{1}(\Theta)=\left[\begin{array}{ll}\mathbf{a}^{T}(\Theta) & \mathbf{0}_{N}^{T}\end{array}\right]^{T}, \mathbf{a}_{2}(\Theta)=\left[\begin{array}{cc}\mathbf{0}_{N}^{T} & \mathbf{a}^{H}(\Theta)\end{array}\right]^{T}$ and $\mathbf{0}_{N}$ is the null vector of size $N, \mathbf{A}(\Theta)=$ $\left[\begin{array}{ll}\mathbf{a}_{3}(\Theta) & \mathbf{a}_{4}(\Theta)\end{array}\right]$ where $\mathbf{a}_{3}(\Theta)=\left[\begin{array}{ll}\mathbf{a}^{T}(\Theta) & \mathbf{a}^{H}(\Theta)\end{array}\right]^{T}$ and $\mathbf{a}_{4}(\Theta)=\left[\begin{array}{ll}j \mathbf{a}^{T}(\Theta) & -j \mathbf{a}^{H}(\Theta)\end{array}\right]^{T}$.

\section{B. Particular case of $k$-rectilinear sources}

If the source $i$ is a $k_{i}$-rectilinear source, $m_{i}(t)$ can be written as [11]

$$
m_{i}(t)=\sum_{j=1}^{k_{i}} v_{i j}(t) \exp \left(j \Phi_{i j}\right)
$$

where the signals $v_{i j}(t)\left(1 \leq j \leq k_{i}\right)$ are real-valued and statistically independent whereas $\Phi_{i j}$ is a phase term. A rectilinear source (BPSK or ASK source) is a 1 -rectilinear source, whereas a QPSK source, a square or a rectangular QAM source are three examples of 2 -rectilinear sources. From (3) we deduce that $\mathbf{m}_{i}(t)$ and $\tilde{\mathbf{m}}_{i}(t)$ can be written as

$$
\begin{aligned}
& \mathbf{m}_{i}(t)=\sum_{j=1}^{k_{i}} v_{i j}(t) \mathbf{e}\left(\Phi_{i j}\right), \quad \tilde{\mathbf{m}}_{i}(t)=\sum_{j=1}^{k_{i}} v_{i j}(t) \tilde{\mathbf{e}}\left(\Phi_{i j}\right) \\
& \mathbf{e}(\Phi)=\left[\begin{array}{c}
\cos (\Phi) \\
\sin (\Phi)
\end{array}\right] \quad \tilde{\mathbf{e}}(\Phi)=\left[\begin{array}{l}
\exp (j \Phi) \\
\exp (-j \Phi)
\end{array}\right]
\end{aligned}
$$

Inserting (4) into (2), it is straightforward to show that $\tilde{\mathbf{x}}(t)$ takes the form

$$
\begin{aligned}
& \tilde{\mathbf{x}}(t)=\sum_{i=1}^{P} \sum_{j=1}^{k_{i}} \tilde{\mathbf{b}}\left(\Theta_{i}, \Phi_{i j}\right) v_{i j}(t)+\tilde{\mathbf{n}}(t) \\
& \tilde{\mathbf{b}}(\Theta, \Phi)=\tilde{\mathbf{A}}(\Theta) \tilde{\mathbf{e}}(\Phi)=\mathbf{A}(\Theta) \mathbf{e}(\Phi)
\end{aligned}
$$

where $\tilde{\mathbf{b}}(\Theta, \Phi)$ is the generic extended steering vector of a rectilinear source. This shows that without noise, $\tilde{\mathbf{x}}(t)$ is spanned by the extended steering vectors $\tilde{\mathbf{b}}\left(\Theta_{i}, \Phi_{i j}\right)(1 \leq$ $\left.j \leq k_{i}\right)(1 \leq i \leq P)$ and this allows straightforward $\mathrm{NC}$ extensions of $2 q-$ MUSIC methods $(q \geq 1)$ from the generic extended steering vector $\tilde{\mathbf{b}}(\Theta, \Phi)$ as done in [11].

\section{Problem formulation}

The first purpose of this paper is to show that whatever the kind and the non-circularity properties of the sources, the signal subspace of the SO statistical matrix of $\tilde{\mathbf{x}}(t)$ has the same algebraic structure as the one obtained for $k$-rectilinear sources. The second purpose of this paper is to show that this result remains valid at the FO for most of the sources of practical interest. These results, completely unknown by the scientific community, allow to use, whatever the kind of sources $(q=1)$ and for most of sources of practical interest $(q=2)$, NC extensions of $2 q$-MUSIC $(1 \leq q \leq 2)$ initially developed for $k$-rectilinear sources such as those presented in [11].

\section{NON-CIRCULAR SECOND-ORDER DF METHODS}

\section{A. Extended Second-Order Statistics}

Most of SO NC DF methods exploit the information contained in the time-averaged correlation matrix of $\tilde{\mathbf{x}}(t)$, defined by $\mathbf{R}_{\tilde{x}}=\left\langle\mathbb{E}\left[\tilde{\mathbf{x}}(t) \tilde{\mathbf{x}}^{H}(t)\right]\right\rangle$, where $\langle$.$\rangle is the time averaging$ operation on a given observation window and $\mathbb{E}[$.$] is the$ expected value operation. Under the assumptions of section II-A, we deduce from (2) that $\mathbf{R}_{\tilde{x}}$ can be written as

$$
\mathbf{R}_{\tilde{x}}=\sum_{i=1}^{P} \mathbf{A}\left(\Theta_{i}\right) \mathbf{R}_{m_{i}} \mathbf{A}^{H}\left(\Theta_{i}\right)+\sigma^{2} \mathbf{I}_{N}
$$

where $\sigma^{2}$ is the noise power per sensor and $\mathbf{R}_{m_{i}}$ is the timeaveraged correlation matrix of $\mathbf{m}_{i}(t)$. As $\mathbf{R}_{m_{i}}$ is a real-valued $(2 \times 2)$ symmetric matrix, its eigen decomposition can be written as

$$
\mathbf{R}_{m_{i}}=\sum_{k=1}^{2} \mu_{i k} \mathbf{e}\left(\Phi_{i k}\right) \mathbf{e}^{T}\left(\Phi_{i k}\right)
$$

where the orthonormal eigenvectors e $\left(\Phi_{i k}\right)(1 \leq k \leq 2)$, such that $\mathbf{e}^{T}\left(\Phi_{i k}\right) \mathbf{e}\left(\Phi_{i k^{\prime}}\right)=\delta\left(k-k^{\prime}\right)$, are associated with the real eigenvalues $\mu_{i k}$ where $\delta($.$) is the Kronecker symbol. For this$ reason, $\Phi_{i 2}=\Phi_{i 1} \pm \pi / 2$. Using (9) into (8), we obtain

$$
\mathbf{R}_{\tilde{x}}=\sum_{i=1}^{P} \sum_{k=1}^{2} \mu_{i k} \tilde{\mathbf{b}}\left(\Theta_{i}, \Phi_{i k}\right) \tilde{\mathbf{b}}^{H}\left(\Theta_{i}, \Phi_{i k}\right)+\sigma^{2} \mathbf{I}_{N}
$$

\section{B. Non-circular second order MUSIC methods}

We deduce from (10) that the signal space of $\mathbf{R}_{\tilde{x}}$ is spanned by the vectors $\tilde{\mathbf{b}}\left(\Theta_{i}, \Phi_{i k}\right)$ associated with the non-zero $\mu_{i k}$ $(1 \leq i \leq P)(1 \leq k \leq 2)$. For each $i$ at least one value of $\mu_{i k}$ is not zero and thus at least one $\tilde{\mathbf{b}}\left(\Theta_{i}, \Phi_{i k}\right)$ is in the signal subspace of $\mathbf{R}_{\tilde{x}}$. According to [11][1], the directions $\Theta_{i}(1 \leq i \leq P)$ can then be estimated by searching for the zeros, over $(\Theta, \Phi)$, of the NC1-MUSIC criterion

$$
J_{1,2}(\Theta, \Phi)=\left(\tilde{\mathbf{b}}^{H}(\Theta, \Phi) \boldsymbol{\Pi}_{2} \tilde{\mathbf{b}}(\Theta, \Phi)\right) /\|\tilde{\mathbf{b}}(\Theta, \Phi)\|^{2}
$$

where $\|\mathbf{u}\|^{2}=\mathbf{u}^{H} \mathbf{u}$ and $\boldsymbol{\Pi}_{2}$ is the orthogonal projector on the noise subspace of $\mathbf{R}_{\tilde{x}}$. Thus the NC1-MUSIC algorithm developed for rectilinear or $k$-rectilinear sources [11] can also be used for arbitrary SO NC sources and is able to process up to $2 N-1$ rectilinear sources from a $2 \mathrm{D}$ search process with respect to $(\Theta, \Phi)$ when $\Theta$ is a scalar, i.e for 1D DF estimation.

\section{NON-CIRCULAR FourTh-ORDER DF METHODS}

\section{A. Extended Fourth-Order Statistics}

FO NC DF methods exploit the information contained in the time-averaged circular FO cumulants of $\tilde{\mathbf{x}}(t)$, defined by $c_{\tilde{\mathbf{x}}, i j k l}=<\operatorname{cum}\left(\tilde{x}_{i}(t), \tilde{x}_{j}(t), \tilde{x}_{k}^{*}(t), \tilde{x}_{l}^{*}(t)\right)>$ for $1 \leq$ $i, j, k, l \leq 2 N$, where $\tilde{x}_{i}(t)$ is the component $i$ of $\tilde{\mathbf{x}}(t)$. These latter entries can be arranged in the $(2 N)^{2} \times(2 N)^{2}$ matrix $\mathbf{C}_{\tilde{\mathbf{x}}}$ in different ways as done in [3] or [11] but it is easy to verify [11] that all these arrangements are equivalent. We then choose the natural arrangement defined by $\mathbf{C}_{\tilde{\mathbf{x}}}(I, J)=c_{\tilde{\mathbf{x}}, i j k l}$ with $I=2 N(i-1)+j$ and $J=2 N(k-1)+l$. Under the 
assumptions of section II-A, we deduce from (2) that $\mathbf{C}_{\tilde{\mathbf{x}}}$ can be written as

$$
\mathbf{C}_{\tilde{\mathbf{x}}}=\sum_{i=1}^{P} \tilde{\mathbf{A}}^{\otimes 2}\left(\Theta_{i}\right) \mathbf{C}_{\tilde{\mathbf{m}}_{i}} \tilde{\mathbf{A}}^{\otimes 2 H}\left(\Theta_{i}\right)
$$

where $\tilde{\mathbf{A}}^{\otimes 2}(\Theta)=\tilde{\mathbf{A}}(\Theta) \otimes \tilde{\mathbf{A}}(\Theta), \otimes$ is the kronecker product and $\mathbf{C}_{\tilde{\mathbf{m}}_{i}}$ is the $(4 \times 4)$ matrix of the time-averaged circular FO cumulants of $\tilde{\mathbf{m}}_{i}(t)$. Denoting by $\beta_{i}$ and $\gamma_{i}$ the parameters: $\beta_{i}=<\operatorname{cum}\left(m_{i}(t), m_{i}(t), m_{i}(t), m_{i}^{*}(t)\right)>/ c_{i}$, $\gamma_{i}=<\operatorname{cum}\left(m_{i}(t), m_{i}(t), m_{i}(t), m_{i}(t)\right)>/ c_{i}$ and $c_{i}=$ $c_{\tilde{\mathbf{m}}_{i}, 1111}$, the matrix $\mathbf{C}_{\tilde{\mathbf{m}}_{i}}$ can be written as $\mathbf{C}_{\tilde{\mathbf{m}}_{i}}=c_{i} \mathbf{C}_{i}$ where $\mathbf{C}_{i}$ is defined by

$$
\mathbf{C}_{i}=\left[\begin{array}{cccc}
1 & \beta_{i} & \beta_{i} & \gamma_{i} \\
\beta_{i}^{*} & 1 & 1 & \beta_{i} \\
\beta_{i}^{*} & 1 & 1 & \beta_{i} \\
\gamma_{i}^{*} & \beta_{i}^{*} & \beta_{i}^{*} & 1
\end{array}\right]=\boldsymbol{\Gamma} \overline{\mathbf{C}}_{i} \boldsymbol{\Gamma}^{H}
$$

where $\boldsymbol{\Gamma}$ and $\overline{\mathbf{C}}_{i}$ are defined by

$$
\boldsymbol{\Gamma}=\left[\begin{array}{lll}
1 & 0 & 0 \\
0 & 1 & 0 \\
0 & 1 & 0 \\
0 & 0 & 1
\end{array}\right] \quad \text { and } \overline{\mathbf{C}}_{i}=\left[\begin{array}{ccc}
1 & \beta_{i} & \gamma_{i} \\
\beta_{i}^{*} & 1 & \beta_{i} \\
\gamma_{i}^{*} & \beta_{i}^{*} & 1
\end{array}\right]
$$

It becomes obvious from (13) that the rank of $\mathbf{C}_{i}$ is at most equal to 3 and the eigen-decomposition of $\mathbf{C}_{\tilde{\mathbf{m}}_{i}}$, Hermitian matrix, can be written as

$$
\mathbf{C}_{\tilde{\mathbf{m}}_{i}}=c_{i} \sum_{j=1}^{3} \mu_{i j} \mathbf{u}_{i j} \mathbf{u}_{i j}^{H}
$$

where the $\mu_{i j}$ 's $(1 \leq j \leq 3)$ are the three real eigenvalues of $\mathbf{C}_{i}$ with the greatest modulus, whereas the $\mathbf{u}_{i j}$ 's are the associated orthonormal eigenvectors. Depending on the source $i$, one, two or three of the $\mu_{i j}$ 's may not be zero. We define a $r_{i}-\operatorname{rank}$ source $i \quad\left(1 \leq r_{i} \leq 3\right)$, a source $i$ for which only $r_{i}$ eigenvalues $\mu_{i j}$ 's $\left(1 \leq j \leq r_{i}\right)$ are not zero.

The purpose of what follows is to show that the space spanned by the vectors $\mathbf{u}_{i j}\left(1 \leq j \leq r_{i}\right)$ is also spanned by vectors of the form $\tilde{\mathbf{e}}^{\otimes 2}\left(\Psi_{i j}\right)\left(1 \leq j \leq r_{i}\right)$. In other words, we will show that $\mathbf{C}_{\tilde{\mathbf{m}}_{i}}$ can also be written as

$$
\mathbf{C}_{\tilde{\mathbf{m}}_{i}}=\sum_{j=1}^{r_{i}} \sum_{l=1}^{r_{i}} \tilde{\mathbf{e}}^{\otimes 2}\left(\Psi_{i j}\right) \tilde{\mathbf{e}}^{\otimes 2 H}\left(\Psi_{i l}\right) q_{j l}^{i}
$$

where the $q_{j l}^{i}$ 's and $\Psi_{i l}$ 's $\left(1 \leq j, l \leq r_{i}\right)$ are scalar quantities.

\section{B. Algebraic structure of $\mathbf{C}_{\tilde{\mathbf{m}}_{i}}$}

We analyse in this section the algebraic structure of $\mathbf{C}_{i}=$ $\mathbf{C}_{\tilde{\mathbf{m}}_{i}} / c_{i}$ for each possible value of its rank $r_{i}$, i.e. for $1 \leq$ $r_{i} \leq 3$.

1) Rank one source $i$ : The matrix $\mathbf{C}_{i}$ (12) has a rank equal to 1 if and only if the determinants of all the $(2 \times 2)$ submatrices of $\mathbf{C}_{i}$ are equal to zero. This is obtained if $\mathbf{C} \mathbf{1}$, defined by (16), is verified

$$
\text { C1 : } \quad\left|\beta_{i}\right|=1 \text { and } \gamma_{i}=\beta_{i}^{2}
$$

In this case, $\exists \Psi_{i 1}$ such that $\beta_{i}=\exp \left(2 j \Psi_{i 1}\right)$ and $\gamma_{i}=$ $\exp \left(4 j \Psi_{i 1}\right)$. It is then easy to verify that in this case $\mathbf{C}_{\tilde{\mathbf{m}}_{i}}$ $=c_{i} \mathbf{C}_{i}$ takes the form

$$
\mathbf{C}_{\tilde{\mathbf{m}}_{i}}=c_{i} \tilde{\mathbf{e}}^{\otimes 2}\left(\Psi_{i 1}\right) \tilde{\mathbf{e}}^{\otimes 2 H}\left(\Psi_{i 1}\right)
$$

which is a particular case of (15) with $q_{11}^{i}=c_{i}$ and $r_{i}=1$.
2) Rank 2 source $i$ : The matrix $\mathbf{C}_{i}$ (12) has a rank equal to 2 if and only if the determinant of $\overline{\mathbf{C}}_{i}$ (13) is equal to zero while $\mathbf{C 1}$ is not verified. After some elementaries computations, the rank 2 condition is obtained if $\mathbf{C 2}$, defined by (17), is verified

$$
\text { C2 : } \exists \Omega_{i} / \gamma_{i}=\left(\beta_{i}\right)^{2}+e^{j \Omega_{i}}\left(\left|\beta_{i}\right|^{2}-1\right) \text { and }\left|\beta_{i}\right| \neq 1
$$

Condition $\mathbf{C 2}$ implies that the matrix $\mathbf{Q}_{i}$ composed of the two first columns of $\mathbf{C}_{i}$ has a rank equal to 2 . Let us analyze the conditions under which there exist $\Psi_{i 1}$ and $\Psi_{i 2}$ such that $\operatorname{Span}\left(\mathbf{Q}_{i}\right)=\operatorname{Span}\left(\tilde{\mathbf{e}}^{\otimes 2}\left(\Psi_{i 1}\right), \tilde{\mathbf{e}}^{\otimes 2}\left(\Psi_{i 2}\right)\right)$. This last property is verified if and only if $\exists\left(\Psi_{i 1}, \Psi_{i 2}\right) \in \mathbb{R}^{2}$ and $\exists \mathbf{T}$, a full rank $(2 \times 2)$ matrix, such that

$$
\mathbf{Q}_{i}=\left[\begin{array}{ll}
1 & \beta_{i} \\
\beta_{i}^{*} & 1 \\
\beta_{i}^{*} & 1 \\
\gamma_{i}^{*} & \beta_{i}^{*}
\end{array}\right]=\left[\begin{array}{l}
\mathbf{Q}_{1}^{i} \\
\mathbf{Q}_{2}^{i}
\end{array}\right]=\left[\begin{array}{l}
\mathbf{E}_{12}^{i} \\
\mathbf{E}_{12}^{i} \Omega_{12}^{i}
\end{array}\right] \mathbf{T}
$$

where the $(2 \times 2)$ matrices $\mathbf{Q}_{j}^{i}, \Omega_{12}^{i}$ and $\mathbf{E}_{12}^{i}$ are defined by

$$
\begin{aligned}
& \mathbf{Q}_{1}^{i}=\left[\begin{array}{ll}
1 & \beta_{i} \\
\beta_{i}^{*} & 1
\end{array}\right] ; \quad \mathbf{Q}_{2}^{i}=\left[\begin{array}{ll}
\beta_{i}^{*} & 1 \\
\gamma_{i}^{*} & \beta_{i}^{*}
\end{array}\right] \\
& \mathbf{E}_{12}^{i}=\left[\begin{array}{ll}
e^{j 2 \Psi_{i 1}} & e^{j 2 \Psi_{i 2}} \\
1 & 1
\end{array}\right] ; \quad \Omega_{12}^{i}=\left[\begin{array}{ll}
e^{-j 2 \Psi_{i 1}} & 0 \\
0 & e^{-j 2 \Psi_{i 2}}
\end{array}\right]
\end{aligned}
$$

After straightforward manipulations, it is easy to verify that property (18) is equivalent to $\mathbf{Q}_{1}^{i}=\mathbf{E}_{12}^{i} \mathbf{T}$ and $\mathbf{T}\left(\mathbf{Q}_{1}^{i}\right)^{-1}$ $\mathbf{Q}_{2}^{i} \mathbf{T}^{-1}=\Omega_{12}^{i}$ which requires that $e^{-j 2 \Psi_{i 1}}$ and $e^{-j 2 \Psi_{i 2}}$ are eigenvalues of $\left(\mathbf{Q}_{1}^{i}\right)^{-1} \mathbf{Q}_{2}^{i}$ and that the associated eigenvectors correspond to the columns of $\mathbf{T}^{-1}$. From (17) and (19), we obtain, after some elementary computations

$$
\begin{gathered}
\left(\mathbf{Q}_{1}^{i}\right)^{-1} \mathbf{Q}_{2}^{i}=\left[\begin{array}{cc}
\alpha_{i} & 1 \\
-e^{-j \Omega_{i}} & 0
\end{array}\right] \\
\text { where } \alpha_{i}=2 \Re\left(\beta_{i} e^{-j \Omega_{i} / 2}\right) e^{-j \Omega_{i} / 2}
\end{gathered}
$$

We then deduce that the eigenvalues, $\lambda_{i k}(1 \leq k \leq 2)$, of (20) are given by

$$
\lambda_{i k}=e^{-j \Omega_{i} / 2}\left(\Re\left(\beta_{i} e^{-j \Omega_{i} / 2}\right) \pm \sqrt{\Re\left(\beta_{i} e^{-j \Omega_{i} / 2}\right)^{2}-1}\right)
$$

It is then easy to verify that $\left|\lambda_{i k}\right|^{2}=1$ for $(1 \leq k \leq 2)$ and $\lambda_{i 1} \neq \lambda_{i 2}$ if condition $\mathbf{C 3}$, defined hereafter, is verified

$$
\text { C3 : }\left|\Re\left(\beta_{i} e^{-j \Omega_{i} / 2}\right)\right| \leq 1
$$

In this case, it exist $\Psi_{i 1}$ and $\Psi_{i 2}$ such that $\lambda_{i 1}=e^{-j 2 \Psi_{i 1}}$ and $\lambda_{i 2}=e^{-j 2 \Psi_{i 2}}$. Moreover, it is also easy to verify that $\mathbf{Q}_{1}^{i}=\mathbf{E}_{12}^{i} \mathbf{T}$, which means that $\mathbf{T}\left(\mathbf{Q}_{1}^{i}\right)^{-1} \mathbf{Q}_{2}^{i} \mathbf{T}^{-1}=\Omega_{12}^{i}$ is verified and that $\operatorname{Span}\left(\mathbf{Q}_{i}\right)=\operatorname{Span}\left(\tilde{\mathbf{e}}^{\otimes 2}\left(\Psi_{i 1}\right), \tilde{\mathbf{e}}^{\otimes 2}\left(\Psi_{i 2}\right)\right)$. Matrix $\mathbf{C}_{\tilde{\mathbf{m}}_{i}}$ then takes the form (15). However if condition C4 is verified

$$
\text { C4 : } \quad\left|\Re\left(\beta_{i} e^{-j \Omega_{i} / 2}\right)\right|>1
$$

the previous results do no longer hold, it does not exist $\Psi_{i 1}$ and $\Psi_{i 2}$ such that $\operatorname{Span}\left(\mathbf{Q}_{i}\right)=\operatorname{Span}\left(\tilde{\mathbf{e}}^{\otimes 2}\left(\Psi_{i 1}\right), \tilde{\mathbf{e}}^{\otimes 2}\left(\Psi_{i 2}\right)\right)$ and $\mathbf{C}_{\tilde{\mathbf{m}}_{i}}$ has no longer the form (15). However, most of rank 2 sources encountered in practice, such as square QAM sources, verify (23) and not (24). 
3) Rank 3 source $i$ : In this case,

$$
\text { C5 : } \quad\left|\gamma_{i}-\left(\beta_{i}\right)^{2}\right| \neq\left.|| \beta_{i}\right|^{2}-1 \mid
$$

the space spanned by the $\mathbf{u}_{i j}$ 's $(1 \leq j \leq 3)$ of (14) corresponds to the one spanned by the columns of $\boldsymbol{\Gamma}$, denoted by $\operatorname{Span}(\boldsymbol{\Gamma})$. It is well-known that the orthogonal projector on the subspace orthogonal to the columns of $\Gamma$ is defined by $\Pi_{\Gamma}^{\perp}=\mathbf{I}-\boldsymbol{\Gamma}\left(\boldsymbol{\Gamma}^{H} \boldsymbol{\Gamma}\right)^{-1} \boldsymbol{\Gamma}^{H}$ where it is easy to verify that $\boldsymbol{\Gamma}^{H} \boldsymbol{\Gamma}=\operatorname{diag}\left(\left[\begin{array}{lll}1 & 2 & 1\end{array}\right]\right)$. Then a vector $\mathbf{v}$ belongs to $\operatorname{Span}(\boldsymbol{\Gamma})$ if and only if $\Pi_{\Gamma}^{\perp} \mathbf{v}=0$. It is then straightforward to verify that, whatever the value of $\Psi, \Pi_{\Gamma}^{\perp} \tilde{\mathbf{e}}^{\otimes 2}(\Psi)=0$, which means that all vectors $\tilde{\mathbf{e}}^{\otimes 2}(\Psi)$ for arbitrary values of $\Psi$ belong to $\operatorname{Span}(\boldsymbol{\Gamma})$. Moreover, it is easy to built three non-colinear vectors $\tilde{\mathbf{e}}^{\otimes 2}\left(\Psi_{i j}\right)(1 \leq j \leq 3)$ where $\Psi_{i 1} \neq \Psi_{i 2} \neq \Psi_{i 3}$, which shows that there exists $\tilde{\mathbf{e}}^{\otimes 2}\left(\Psi_{i j}\right)(1 \leq j \leq 3)$ such that $\operatorname{Span}(\boldsymbol{\Gamma})=\operatorname{Span}\left(\tilde{\mathbf{e}}^{\otimes 2}\left(\Psi_{i j}\right), 1 \leq j \leq 3\right)$ and then such that (15) holds.

\section{Non-circular Fourth order MUSIC methods}

It has been shown in section IV-B that in the presence of a mixture of $P$ sources $i(1 \leq i \leq P)$ with either rank 1 , rank 2 verifying (23) or rank $3, \mathbf{C}_{\tilde{\mathbf{m}}_{i}}$ has, for each $i$, the form (15). Inserting (15) into (11) and using (7) we finally obtain

$$
\mathbf{C}_{\tilde{\mathbf{x}}}=\sum_{i=1}^{P} \sum_{j, l=1}^{r_{i}} q_{j l}^{i} \tilde{\mathbf{b}}^{\otimes 2}\left(\Theta_{i}, \Psi_{i j}\right) \tilde{\mathbf{b}}^{\otimes 2 H}\left(\Theta_{i}, \Psi_{i l}\right)
$$

We deduce from (26) that the signal space of $\mathbf{C}_{\tilde{\mathbf{x}}}$ is spanned by the vectors $\tilde{\mathbf{b}}^{\otimes 2}\left(\Theta_{i}, \Psi_{i j}\right)$ for $(1 \leq i \leq P)\left(1 \leq j \leq r_{i}\right)$. For each $i$ at least one vector $\tilde{\mathbf{b}}^{\otimes 2}\left(\Theta_{i}, \Psi_{i j}\right)$ is in the signal subspace of $\mathbf{C}_{\tilde{\mathbf{x}}}$ of rank $r=\sum_{i=1}^{P} r_{i}$. The directions $\Theta_{i}(1 \leq$ $i \leq P$ ) can then be estimated by searching for the zeros or the minima, over $(\Theta, \Psi)$, of the $N C 1-4-$ MUSIC [11] criterion.

$J_{1,4}(\Theta, \Phi)=\left(\tilde{\mathbf{b}}^{\otimes 2 H}(\Theta, \Phi) \boldsymbol{\Pi}_{4} \tilde{\mathbf{b}}^{\otimes 2}(\Theta, \Phi)\right) /\left\|\tilde{\mathbf{b}}^{\otimes 2}(\Theta, \Phi)\right\|^{2}$ where $\Pi_{4}$ is the orthogonal projector on the noise subspace of $\mathbf{C}_{\tilde{\mathbf{x}}}$. For rank 2 sources verifying (24), the $N C 2-4$-MUSIC method presented in [11] must be used.

\section{Computer Simulations}

To illustrate the performance of the $N C 1-2 q$-MUSIC method for $q=1,2$, we consider a mixture of $P=2$ statistically independent sources, having the same SNR equal to $10 \mathrm{~dB}$, impinging on a uniform circular array of $N=3$ antennas of radius $\lambda / 2$, where $\lambda$ is the wavelength. The first source is a 2 -rectilinear source (rank 2 ) whereas the second one is an ASK source (rank 1). The angles of arrival of the 2 sources are $\Theta_{1}=100^{\circ}$ and $\Theta_{2}=110^{\circ}$, whereas their phase are $\Psi_{11}=10^{\circ}, \Psi_{12}=80^{\circ}$ and $\Psi_{21}=45^{\circ}$. Under these assumptions, Fig. 1 shows the variations of the Root Mean Square Error (RMSE) of the direction estimate of the source 1 as a function of the number of snapshots $L$ used to estimate the statistics for both $2 q-$ MUSIC and $N C 1-2 q-$ MUSIC with $q=1$ and 2 . Note the best performance of $N C 1-2 q-$ MUSIC with respect to $2 q-$ MUSIC for both $q=1$ and 2 and the better performance of $\mathrm{HO}$ methods since the sources are poorly angularly separated.

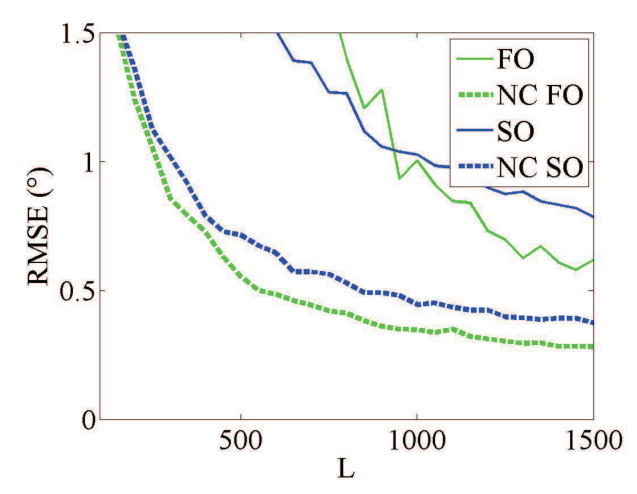

Fig. 1. RMSE of the source 1 as a function of $L$ for $2 q-$ MUSIC and $N C 1-2 q-$ MUSIC with $q=1,2$.

\section{CONCLUSION}

It has been shown in this paper that the $N C 1-2 q$-MUSIC algorithm developed in [11] for $k$-rectilinear sources is also powerful for arbitrary NC sources for $q=1$ and for rank 1, rank 3 and most of rank $2 \mathrm{NC}$ sources encountered in practice for $q=2$.

\section{REFERENCES}

[1] R. O. Schmidt, "Multiple emitter location and signal parameter estimation," IEEE Transactions on AP, vol. 34, no. 3, pp. 276-280, 1986.

[2] B. Porat and B. Friedlander, "Direction finding algorithms based on higher order statistics," IEEE Trans. Signal Processing, vol. 39, no. 9, pp. 2016-2024, Sept. 1991.

[3] P. Chevalier, A. Ferréol, and L. Albera, "High resolution direction finding from higher order statistics : The 2Q-MUSIC algorithm," IEEE Trans. Signal Processing, vol. 54, no. 8, pp. 2986-2997, Aug. 2006.

[4] P. Gounon, C. Adnet, and G.Galy, "Angular Localisation for Non Circular signals," Traitement du signal, vol. 15, no. 1, pp. 17-23, 1998.

[5] P. Chargé, Y. Wang, and J. Saillard, "A non circular sources direction finding method using polynomial rooting," Signal.Processing, vol. 81, no. 8, pp. 1765-1770, Aug. 2001.

[6] F. Gao, A. Nallanathan, and Y. Wang, "Improved MUSIC under the coexistence of both circular and noncircular sources," IEEE Trans. Signal Processing, vol. 56, no. 7, pp. 3033-3038, July 2008.

[7] P. Chargé, Y. Wang, and J. Saillard, "An extended cyclic MUSIC algorithm," IEEE Trans. Signal Processing, vol. 51, no. 7, pp. 17651770 , July 2003.

[8] H. Abeida and J. Delmas, "MUSIC-like Estimation of Direction of Arrival for Noncircular sources," IEEE Trans. Signal Processing, vol. 54, no. 7, pp. 2678-2690, July 2006.

[9] X. Gou, Z. Liu, and Y. Xu, "Biquaternion cumulant-MUSIC for DOA estimation of noncircular signals," Signal.Processing, vol. 93, pp. 874881, 2013.

[10] J.Liu, Z.Huang, and Y.Zhou, "Extended 2q-music algorithm for noncircular signals," Signal.Processing, vol. 88, no. 6, pp. 1327-1339, June 2008.

[11] A. Ferréol and P. Chevalier, "Higher order direction finding for arbitrary non circular sources : The NC-2q-music algorithm," in EUSIPCO, Aalborg, Aug. 2010.

[12] A. Ferréol, E. Boyer, and P. Larzabal, "A low cost algorithm for some bearing estimation methods in the presence of separable nuisance parameters," Electronic.Letters, vol. 40, no. 15, pp. 966-967, July 2004. 